\title{
A FAST SEGMENTATION ALGORITHM FOR C-V MODEL BASED ON EXPONENTIAL IMAGE SEQUENCE GENERATION
}

\author{
Jiaojing $\mathrm{HU}^{\mathrm{a}, \mathrm{b},}$, Lijun $\mathrm{LU}^{\mathrm{b}}$, Junyi XU ${ }^{\mathrm{a}, *}$, Jixian Zhang ${ }^{\mathrm{b}}$ \\ ${ }^{a}$ College of Geomatics, Shandong University of Science and Technology, Qingdao 266510,China -rshujj@163.com \\ ${ }^{\mathrm{b}}$ Chinese Academy of Surveying \& Mapping, Beijing 100830, China -xjyxxl@ sina.com
}

Commission III, WG III/6

KEY WORDS: SAR, coastline, image sequence generation, exponential C-V model

\begin{abstract}
:
For the island coastline segmentation, a fast segmentation algorithm for $\mathrm{C}-\mathrm{V}$ model method based on exponential image sequence generation is proposed in this paper. The exponential multi-scale $\mathrm{C}-\mathrm{V}$ model with level set inheritance and boundary inheritance is developed. The main research contributions are as follows: 1) the problems of the "holes" and "gaps" are solved when extraction coastline through the small scale shrinkage, low-pass filtering and area sorting of region. 2) the initial value of SDF (Signal Distance Function) and the level set are given by Otsu segmentation based on the difference of reflection SAR on land and sea, which are finely close to the coastline. 3) the computational complexity of continuous transition are successfully reduced between the different scales by the SDF and of level set inheritance. Experiment results show that the method accelerates the acquisition of initial level set formation, shortens the time of the extraction of coastline, at the same time, removes the non-coastline body part and improves the identification precision of the main body coastline, which automates the process of coastline segmentation.
\end{abstract}

\section{INTRODUCTION}

It has become a hot issue in the ocean research to extract coastline information quickly and accurately. The ocean is more and more important on the national economy and accelerates the development of ocean transportation. At home and abroad, coastline extraction method focuses on the study of regional growth method, tracking method, edge detection method, level set method and so on. Region growing algorithm has higher demand for the principles design of the growth. Tracking method needs to set the starting point and human intervention for many times. Edge detection algorithm is sensitive to noise, and the result contains too much other useless edge. Level set method expresses the closed curve by constructing threedimensional curved surface, avoids the closed curve evolution process of tracking, and has good detection effect and advantages of strong anti-noise ability. However, the level set method has complex iterative and large amount of calculation, the initial curve to a given. For huge-size SAR image of coastline detection, detection speed is slow; it takes a long time to obtain the result. Reducing the number of iterations and improving testing efficiency become the focus in the study of this paper, guaranteeing the accuracy of the results under the premise. Therefore, this paper proposes an exponential multiscale $\mathrm{C}-\mathrm{V}$ model method to improve efficiency.

\section{TITLE AND ABSTRACT BLOCK}

\subsection{Spots' Eliminate under Multi-scale}

SAR image has speckle noise, factors of reef, fisheries and other factors. These factors can not be removed by the simple lowpass filtering. Exponential multi-scale $\mathrm{C}-\mathrm{V}$ model reduces plaque by narrowing the scale and reducing the resolution, which makes the initial coastline boundary much easier to be obtained. There are many kinds of methods forming image sequence from low spatial resolution to the high spatial resolution by re-sampling. Both images re-sampling based on Shannon sampling theorem and high-low frequency of wavelet multi-resolution decomposition that based on the wavelet theory are typical decomposition approach. We need relatively continuous changes of image sequences on the spatial resolution to ensure the differences between adjacent images in the amount of information, and ensure the robustness of boundary inheritance and continuity. The re-sampling based on Shannon sampling theorem is able to recovery information completely, which is not in conformity with the principle of image sequence difference. There are striking differences between image sequences of wavelet decomposition, which doesn't conform to the principle of robustness and continuity. Due to make the step be smaller than the step of binary wavelet and the sampling step on demand, we use Bessel cubic spline interpolation, also so as to make the sampling step larger than the given step of Shannon sampling theorem (According to the source frequency, complex images' step size always be set to 1 , which also be the original images). The row and column interpolation formulas are:

$$
s_{i j}^{(2)}(y)=\frac{1}{12}\left(\begin{array}{llll}
t^{3} & t^{2} & t & 1
\end{array}\right)\left(\begin{array}{cccccc}
1 & -7 & 16 & -16 & 7 & -1 \\
-2 & 15 & -28 & 20 & -6 & 1 \\
1 & -8 & 0 & 8 & -1 & 0 \\
0 & 0 & 12 & 0 & 0 & 0
\end{array}\right)\left(\begin{array}{c}
z_{i, j-2} \\
z_{i, j-1} \\
z_{i, j} \\
z_{i, j+1} \\
z_{i, j+2} \\
z_{i, j+3}
\end{array}\right)
$$

\footnotetext{
* Corresponding author
} 


$$
s_{i j}^{(1)}(x)=\frac{1}{12}\left(\begin{array}{llll}
3 & s^{2} & s & 1
\end{array}\right)\left(\begin{array}{cccccc}
1 & -7 & 16 & -16 & 7 & -1 \\
-2 & 15 & -28 & 20 & -6 & 1 \\
1 & -8 & 0 & 8 & -1 & 0 \\
0 & 0 & 12 & 0 & 0 & 0
\end{array}\right)\left(\begin{array}{c}
z_{i-2, j} \\
z_{i-1, j} \\
z_{i, j} \\
z_{i+1, j} \\
z_{i+2, j} \\
z_{i+3, j}
\end{array}\right)
$$

Where $s=\left(x-x_{i}\right) /\left(x_{i+1}-x_{i}\right), t=\left(y-y_{j}\right) /\left(y_{j+1}-y_{j}\right)$, $x_{i} \leq x \leq x_{i+1}, y_{j} \leq y \leq x_{j+1}$. For images, we can make $x_{i}=i, y_{j}=j$. The data for the length of $\mathrm{n}$ that is given by the sampling step $\mathrm{T}$, and sampling point location is:

$$
\begin{gathered}
x(u)=1+T^{*} u, 0 \leq u \leq \text { floor }((m-1) / T)+1 \\
y(v)=1+T^{*} v, 0 \leq v \leq \text { floor }((n-1) / T)+1 \\
(i, j)=(1,1),(1,2), \ldots,(m, n)
\end{gathered}
$$

In addition to solve the boundary calculation problem, this paper uses the mirror symmetry extension to expand the image boundary. Extension to the four image corners uses final extended results, which does not affect the calculation of nonexpansion. The calculation of two-dimensional image uses tensor product form of one-dimensional Bessel cubic spline interpolation:

$$
s_{i j}(x, y)=\sqrt{s_{i j}^{(1)}(x) * s_{i j}^{(2)}(y)}
$$

There are also some spots or patches in the residual image sequences of different scales. As a result, we use Butter-worth low-pass filter:

$$
H(u, v)=\frac{1}{1+(\sqrt{2}-1)\left[\frac{D(\mathrm{u}, v)}{D_{0}}\right]^{2 \mathrm{n}}}
$$

\subsection{C-V Model}

$\mathrm{C}-\mathrm{V}$ model is one of the most intensively researched level set algorithms [4]. It streamlines Mumford-Shah model, uses level set theory, evolves curve by minimizing the energy function. The energy function of the model can be expressed as:

$$
\begin{aligned}
& E(u, C)=\int_{\Omega}\left|u^{+}-u_{0}\right|^{2} d x d y \\
& +\mu \int_{\Omega}|\nabla u|^{2} d x d y+v * \text { length }(C)
\end{aligned}
$$

Using level set function $\varphi(x, y, t)$ instead of the curve $C(t)$, there will be the Heaviside function of $H(\varphi)=1, \varphi \geq 0 ; H(\varphi)=1, \varphi \leq 0 ;$ together with the function of $\delta(\varphi)=d H(\varphi) / d \varphi$. The simplified expression of the energy function is:

$$
\begin{aligned}
& E\left(u^{+}, u^{-}, \varphi\right)=\int_{\Omega}\left|u^{+}-u_{0}\right|^{2} H(\varphi) d x d y \\
& +\mu \int_{\Omega}\left|\nabla u^{+}\right|^{2} H(\varphi) d x d y+ \\
& \int_{\Omega}\left|u^{-}-u_{0}\right|^{2}(1-H(\varphi)) d x d y+ \\
& \mu \int_{\Omega}\left|\nabla u^{-}\right|^{2}(1-H(\varphi)) d x d y+v \int_{\Omega}(\nabla H(\varphi)) d x d y
\end{aligned}
$$

Where partial differential equations related to are derived by Chan and Vese's using of Eulerian-Lagrangian method. The formulae are:

$$
\begin{aligned}
& u^{+}-u_{0}=\mu \Delta u^{+}, \varphi>0 \\
& \partial u^{+} / \partial n=0, \varphi=0 \\
& u^{-}-u_{0}=\mu \Delta u^{-}, \varphi<0 \\
& \partial u^{-} / \partial n=0, \varphi=0 \\
& \partial \varphi / \partial t=\delta(\varphi)[v \nabla(\nabla \varphi / \nabla \varphi \mid)- \\
& \left.\left|u^{+}-u_{0}\right|^{2}-\mu\left|\nabla u^{+}\right|^{2}\right]+\left[\left|u^{-}-u_{0}\right|^{2}+\mu\left|\nabla u^{-}\right|^{2}\right]
\end{aligned}
$$

\subsection{Boundary Extraction Process}

The level set segmentation process of coastline by fast and exponential Multi-scale C-V model is shown as fig.1.

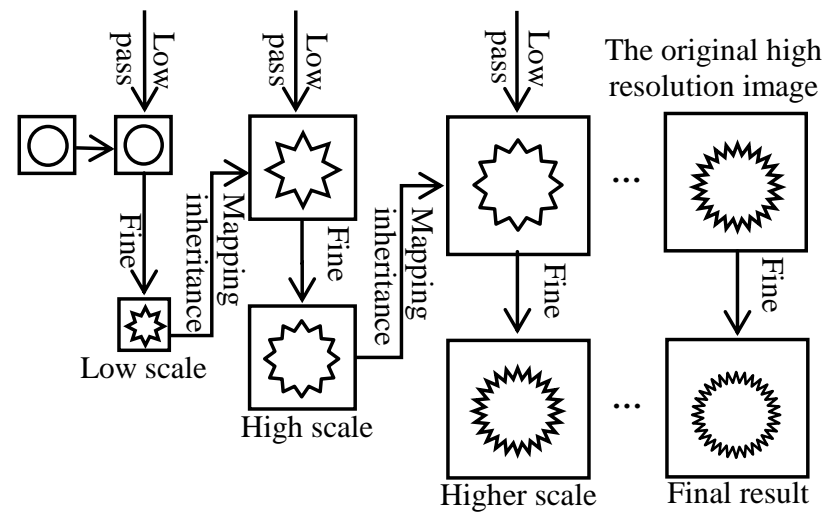

Figure 1. The flowchart of exponential multi-scale $\mathrm{C}-\mathrm{V}$ model of level set.

For the exponential multi-scale C-V model, Multi-scale method realizes image conversion from low scale to high scale; Low pass filter removes the small and pointless feature information, which makes improves the level set $\mathrm{C}-\mathrm{V}$ model to achieve a more simple iterative, greatly shortens time, and refines the boundary. The whole process ensures the efficiency, the high precision of edge extraction and the fast running time.

\section{EXPERIMENTS AND ANALYSIS}

\subsection{The Testing Data}

To evaluate the methodology proposed above, the CosmoSkyMed data images are chosen as test data. The test area locates in the zhoushan of Zhejiang province in China. The Cosmo-SkyMed data images most likely to be used operationally are: 1) they are small islands of the zhoushan Archipelago; 3) resolution $15 \mathrm{~m}$; 4) polarizations VH. As is shown in figure 2 and figure 3 , we select two islands of different position for coastline detection experiments. 

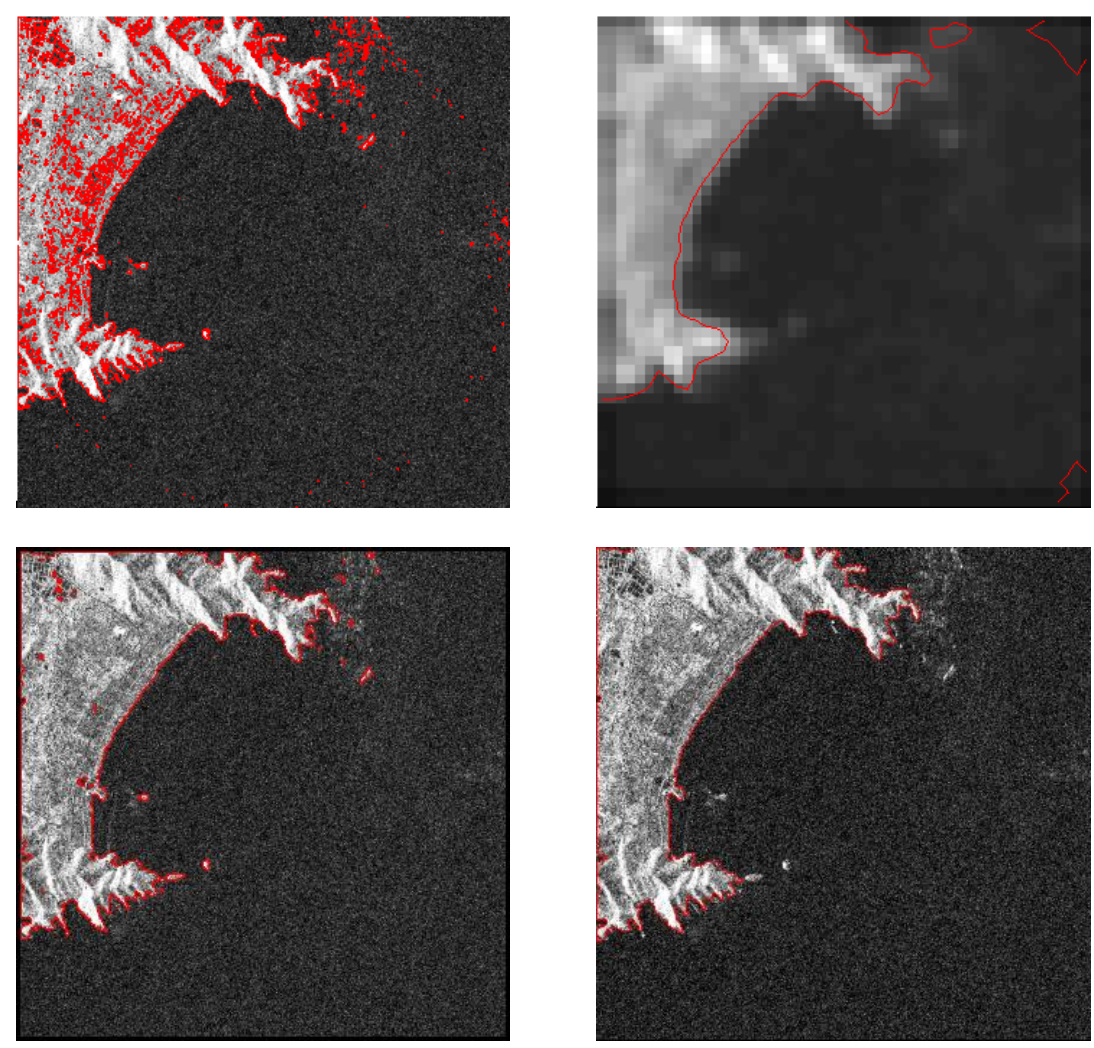

Figure 2. The coastline segmentation results of SAR image: (a) ordinary C-V model result, (b) low scale image sequence result, (c) exponential multi-scale $\mathrm{C}-\mathrm{V}$ model result, (d) automated coastline segmentation result
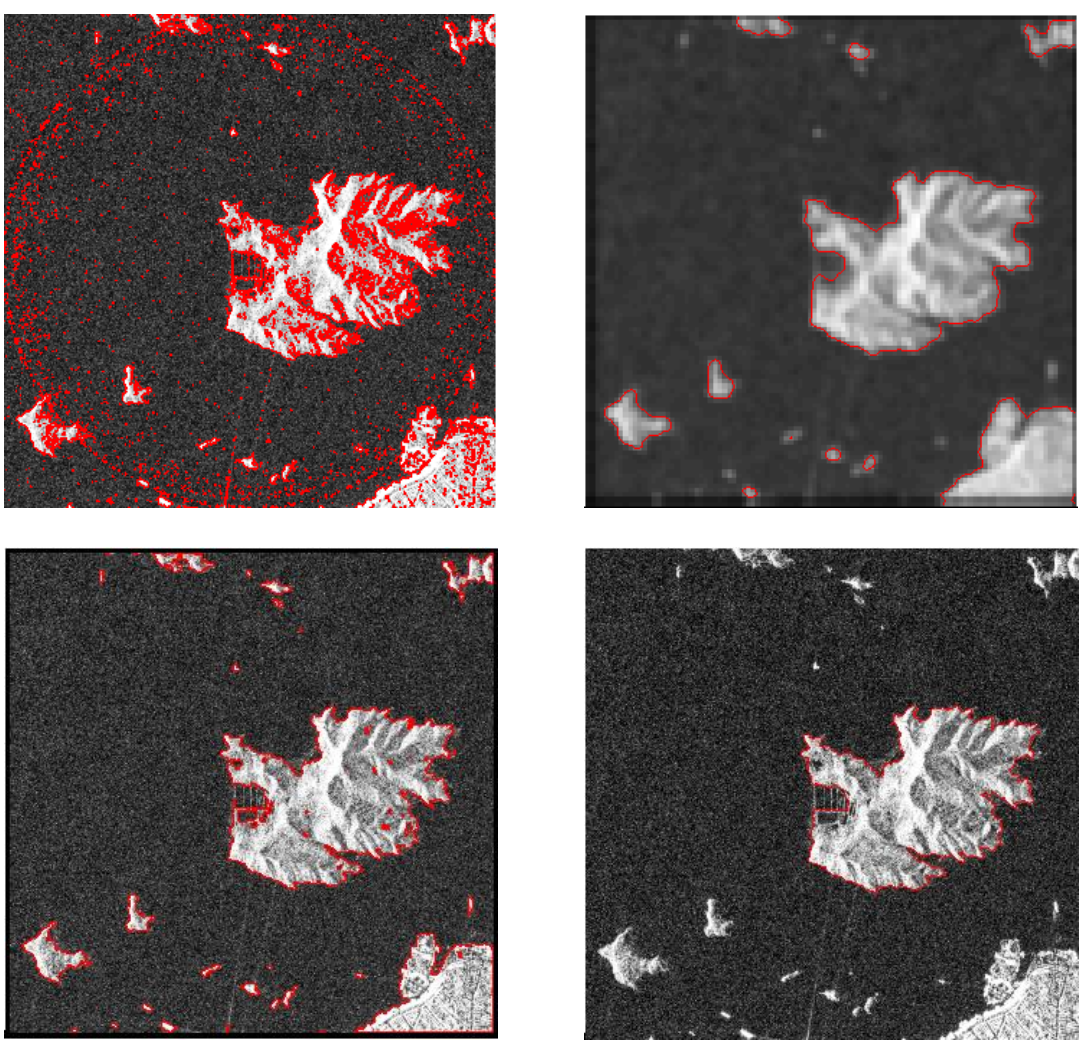

Figure 3. The coastline segmentation results of SAR image: (a) ordinary C-V model result, (b) low scale image sequence result, (c) exponential multi-scale $\mathrm{C}-\mathrm{V}$ model result, (d) automated coastline segmentation result 


\begin{tabular}{|c|c|c|}
\hline Image(1024*1024) & figure 1 & figure 2 \\
\hline ordinary C-V model & $15000 / 6932.78$ & $20000 / 10800.34$ \\
exponential multi-scale C-V model & $8000 / 2160.33$ & $10000 / 3459.87$ \\
\hline
\end{tabular}

Table.1 The number of iterations/time(s) of ordinary C-V model and exponential multi-scale C-V model

\subsection{The Results of Analysis}

To compare the ordinary $\mathrm{C}-\mathrm{V}$ model and exponential multiscale $\mathrm{C}-\mathrm{V}$ model, we set the step as 0.01 . The number of iterations and running time are obtained when we detect the accurate coastline for both the two models

As shown in Table 1, exponential multi-scale $\mathrm{C}-\mathrm{V}$ model reduces the number of iterations and shortens the time. Due to the improper choice of initial contour, the traditional $\mathrm{C}-\mathrm{V}$ model has wrong coastline segmentation phenomenon. If we want to have high-precision, we should increase the step. However, this will cause low convergence rate. exponential multi-scale $\mathrm{C}-\mathrm{V}$ model distinguishes noncoastal fisheries factors by using multi-scale segmentation, which obtains a smaller minimum area and be easier to obtain the precise boundary.

\section{CONCLUSION}

This paper presents a fast and high precision level set exponential multi-scale $\mathrm{C}-\mathrm{V}$ model algorithm for coastline extraction with SAR imagery. The method optimizes the extraction algorithm of huge-size SAR image coastline, which makes it simple for practical engineering. The combination of multi-scale technology and the exponential multi-scale C-V model ensures the high efficiency and accuracy of the segmentation results. Compared with the traditional $\mathrm{C}-\mathrm{V}$ model, exponential multi-scale $\mathrm{C}-\mathrm{V}$ model has higher processing efficiency and less number of iterations, which is more suitable at the same precision level. It is found that the fast level set method evades the partial texture information of land effectively, and omits the SAR coastline segmentation procedure for removing speckle noise. It also has high potentials for the edge extraction of the high resolution image, because of the stronger robustness, convergence and practicability of exponential multiscale $\mathrm{C}-\mathrm{V}$ model.

\section{ACKNOWLEDGEMENT}

This work was supported in part by the National Administration of Surveying, Mapping and Geoinformation (201512022).

\section{REFERENCES}

C.X. Yu, J.Y. Wang, and J. Xu, 2014. "Advance of Coastline Extraction Technology," Journal of Geomatics Science and Technology, 31(3), pp. 305-309.

R.T. Chen, W.H. Xu, and J.Y. Wu, 2012. "New colorful images segmentation algorithm based on level set," Journal of Computer Applications, 32(3), pp.749-751.
Q. Hu, F. Zhang, and R.Y. Liu, et al, 2013. "Land-sea boundary extraction based on gradient vector flow and active contour model," Journal of Zhejiang University (Science Edition), 40(6), pp. 698-704.

X.H. Wang, X. Zhou, and L.L. Fang , 2015.“Segmentation of hyperspectral remote sensing image using vector C-V model," Journal of Remote Sensing, 19(3), PP. 443-450. 